\title{
Fitting the Smallest Enclosing Bregman Ball
}

\author{
Richard Nock ${ }^{1}$ and Frank Nielsen ${ }^{2}$ \\ 1 Université Antilles-Guyane \\ rnock@martinique.univ-ag.fr \\ 2 Sony Computer Science Laboratories, Inc. \\ Frank.Nielsen@acm.org
}

\begin{abstract}
Finding a point which minimizes the maximal distortion with respect to a dataset is an important estimation problem that has recently received growing attentions in machine learning, with the advent of one class classification. We propose two theoretically founded generalizations to arbitrary Bregman divergences, of a recent popular smallest enclosing ball approximation algorithm for Euclidean spaces coined by Bădoiu and Clarkson in 2002.
\end{abstract}

\section{Introduction}

Consider the following problem: given a set of observed data $\mathcal{S}$, compute some accurate set of parameters, or simplified descriptions, that summarize ("fit well") $\mathcal{S}$ according to some criteria. This problem is well known in various fields of statistics and computer science. In many cases, it admits two different formulations:

(1.) Find a point $\mathbf{c}^{*}$ which minimizes an average distortion with respect to $\mathcal{S}$.

(2.) Find a point $\mathbf{c}^{*}$ which minimizes a maximal distortion with respect to $\mathcal{S}$.

These two problems are cornerstones of different subfields of applied mathematics and computer science, such as (i) parametric estimation and the computation of exhaustive statistics for broad classes of distributions in statistics, (ii) one class classification and clustering in machine learning, (iii) the one center problem and its generalizations in computational geometry, among others [1, 2,5, 7]. The main unknown in both problems is what we mean by distortion.

In fact, many examples of distortion measures found in domains concerned by the problems above (computational geometry, machine learning, signal processing, probabilities and statistics, among others) fall into a single family of distortion measures known as Bregman divergences [3. Informally, each of them is the tail of the Taylor expansion of a strictly convex function. Using a neat result in [2, it can be shown that the solution to problem (1.) above is always the average member of $\mathcal{S}$, regardless of the Bregman divergence. This means that problem (1.) can be solved in optimal linear time / space in the size of $\mathcal{S}$ : since $\mathcal{S}$ may be huge, this property is crucial. Unfortunately, the solution of (2.) does not seem to be as affordable; tackling the problem with quadratic programming buys an expensive time complexity cubic in the worst case, and the space complexity is quadratic [8. Notice also that it is mostly used with $L_{2}^{2}$. Instead of 


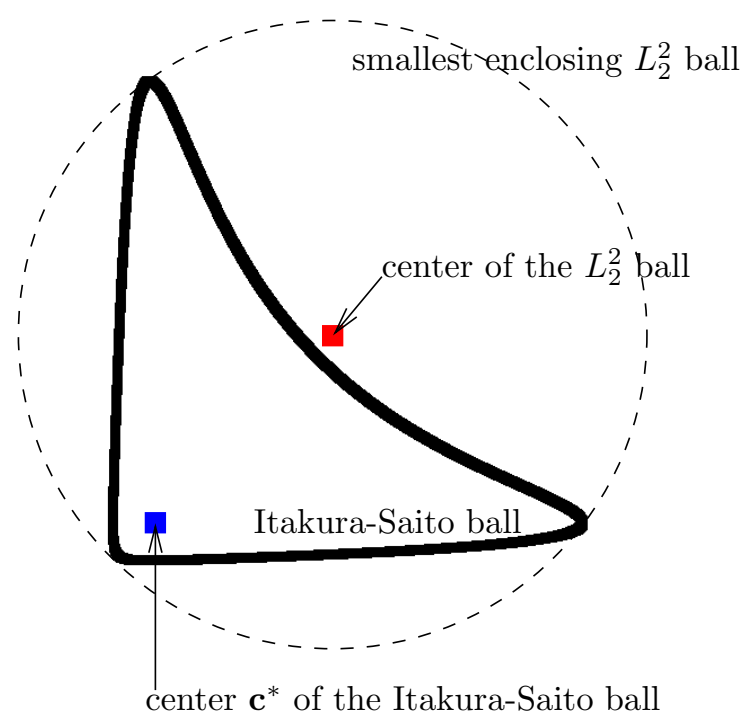

Fig. 1. An optimal Itakura-Saito ball and its smallest enclosing $L_{2}^{2}$ ball, for $d=2$. Notice the poor quality of this optimal approximation: the center of the $L_{2}^{2}$ ball does not even lie inside the Itakura-Saito ball.

finding an exact solution, a recent approach due to [1] approximates the solution of the problem for $L_{2}^{2}$ : the user specifies some $\varepsilon>0$, and the algorithm returns, in time linear in the size of $\mathcal{S}$ (quadratic in $1 / \varepsilon$ ) and in space linear in the size of $\mathcal{S}$, the center $\mathbf{c}$ of a ball which is at $L_{2}^{2}$ divergence no more than $\varepsilon^{2} r^{*}$ from $\mathbf{c}^{*}$. Here, $r^{*}$ is the squared radius of the so-called smallest enclosing ball of $\mathcal{S}$, whose center $\mathbf{c}^{*}$ is obviously the solution to problem (2.). Let us name this algorithm the Bădoiu-Clarkson algorithm, and abbreviate it BC. The key point of the algorithm is its simplicity, which deeply contrasts with quadratic programming approaches: basically, after having initialized $\mathbf{c}$ to a random point of $\mathcal{S}$, we iterate through finding the farthest point away from the current center, and then move along the line between these two points. The popularity of the algorithm, initially focused in computational geometry, has begun to spread to machine learning as well, with its adaptation to fast approximations of SVM training [8].

The applications of $\mathrm{BC}$ have remained so far focused on $L_{2}^{2}$, yet the fact that the algorithm gives a clean and simple approach to problem (2.) for one Bregman divergence naturally raises the question of whether it can be tailored to approximating problem (2.) for any Bregman divergence as well. Figure 1 highlights the importance of this issue.

In this paper, we propose two theoretically founded generalizations of BC to arbitrary Bregman divergences, along with a bijection property that has a flavor similar to a Theorem of 2]: we show a bijection between the set of Bregman divergences and the set of the most commonly used functional averages, which yields that each element of the latter set encodes the minimax distortion solution 
for a Bregman divergence. This property is the cornerstone of our modifications to BC. The next Section presents some definitions. Section 3 gives the theoretical foundations and Section 4 the experiments regarding our generalization of BC.

\section{Definitions}

Our notations mostly follow those of [1,2]. Bold faced variables such as $\mathbf{x}$ and $\boldsymbol{\alpha}$, represent column vectors. Sets are represented by calligraphic upper-case alphabets, e.g. $\mathcal{S}$, and enumerated as $\left\{\mathbf{s}_{i}: i \geq 1\right\}$ for vector sets, and $\left\{s_{i}: i \geq 1\right\}$ otherwise. The $j^{\text {th }}$ component of vector $\mathbf{s}$ is noted $s_{j}$, for $j \leq 1$. Vectors are supposed $d$-dimensional. We write $\mathbf{x} \geq \mathbf{y}$ as a shorthand for $x_{i} \geq y_{i}, \forall i$. The cardinal of a set $\mathcal{S}$ is written $|\mathcal{S}|$, and $\langle.,$.$\rangle defines the inner product for real valued$ vectors, i.e. the dot product. Norms are $L_{2}$ for a vector, and Frobenius for a matrix. Bregman divergences are a parameterized family of distortion measures: let $F: \mathcal{X} \rightarrow \mathbb{R}$ be strictly convex and differentiable on the interior $\operatorname{int}(\mathcal{X})$ of some convex set $\mathcal{X} \subseteq \mathbb{R}^{d}$. Its corresponding Bregman divergence is:

$$
D_{F}\left(\mathbf{x}^{\prime}, \mathbf{x}\right)=F\left(\mathbf{x}^{\prime}\right)-F(\mathbf{x})-\left\langle\mathbf{x}^{\prime}-\mathbf{x}, \nabla_{F}(\mathbf{x})\right\rangle .
$$

Here, $\nabla_{F}$ is the gradient operator of $F$. A Bregman divergence has the following properties: it is convex in $\mathbf{x}^{\prime}$, always non negative, and zero iff $\mathbf{x}=\mathbf{x}^{\prime}$. Whenever $F(\mathbf{x})=\sum_{i=1}^{d} x_{i}^{2}=\|\mathbf{x}\|_{2}^{2}$, the corresponding divergence is the squared Euclidean distance $\left(L_{2}^{2}\right): D_{F}\left(\mathbf{x}^{\prime}, \mathbf{x}\right)=\left\|\mathbf{x}-\mathbf{x}^{\prime}\right\|_{2}^{2}$, with which is associated the common definition of a ball in an Euclidean metric space:

$$
\mathcal{B}_{\mathbf{c}, r}=\left\{\mathbf{x} \in \mathcal{X}:\|\mathbf{x}-\mathbf{c}\|_{2}^{2} \leq r\right\},
$$

with $\mathbf{c} \in \mathcal{S}$ the center of the ball, and $r \geq 0$ its (squared) radius. Eq. (2) suggests a natural generalization to the definition of balls for arbitrary Bregman divergences. However, since a Bregman divergence is usually not symmetric, any $\mathbf{c} \in \mathcal{S}$ and any $r \geq 0$ define actually two dual Bregman balls:

$$
\begin{aligned}
& \mathcal{B}_{\mathbf{c}, r}=\left\{\mathbf{x} \in \mathcal{X}: D_{F}(\mathbf{c}, \mathbf{x}) \leq r\right\} \\
& \mathcal{B}_{\mathbf{c}, r}^{\prime}=\left\{\mathbf{x} \in \mathcal{X}: D_{F}(\mathbf{x}, \mathbf{c}) \leq r\right\} .
\end{aligned}
$$

Remark that $D_{F}(\mathbf{c}, \mathbf{x})$ is always convex in $\mathbf{c}$ while $D_{F}(\mathbf{x}, \mathbf{c})$ is not always, but the boundary $\partial \mathcal{B}_{\mathbf{c}, r}$ is not always convex (it depends on $\mathbf{x}$, given $\mathbf{c}$ ), while $\partial \mathcal{B}_{\mathbf{c}, r}^{\prime}$ is always convex. In this paper, we are mainly interested in $\mathcal{B}_{\mathbf{c}, r}$ because of the convexity of $D_{F}$ in $\mathbf{c}$. The conclusion of the paper extends some results to build $\mathcal{B}_{\mathbf{c}, r}^{\prime}$ as well. Let $\mathcal{S} \subseteq \mathcal{X}$ be a set of $m$ points that were sampled from $\mathcal{X}$. A smallest enclosing Bregman ball (SEBB) for $\mathcal{S}$ is a Bregman ball $\mathcal{B}_{\mathbf{C}^{*}, r^{*}}$ with $r^{*}$ the minimal real such that $\mathcal{S} \subseteq \mathcal{B}_{\mathbf{C}^{*}, r^{*}}$. With a slight abuse of language, we will refer to $r^{*}$ as the radius of the ball. Our objective is to approximate as best as possible the $\mathrm{SEBB}$ of $\mathcal{S}$, which amounts to minimizing the radius of the enclosing ball we build. As a simple matter of fact indeed, the SEBB is unique.

Lemma 1. The smallest enclosing Bregman ball $\mathcal{B}_{\boldsymbol{c}^{*}, r^{*}}$ of $\mathcal{S}$ is unique.

(proof omitted due to the lack of space) Algorithm 1 presents Bădoiu-Clarkson's algorithm for the SEBB approximation problem with the $L_{2}^{2}$ divergence [1]. 


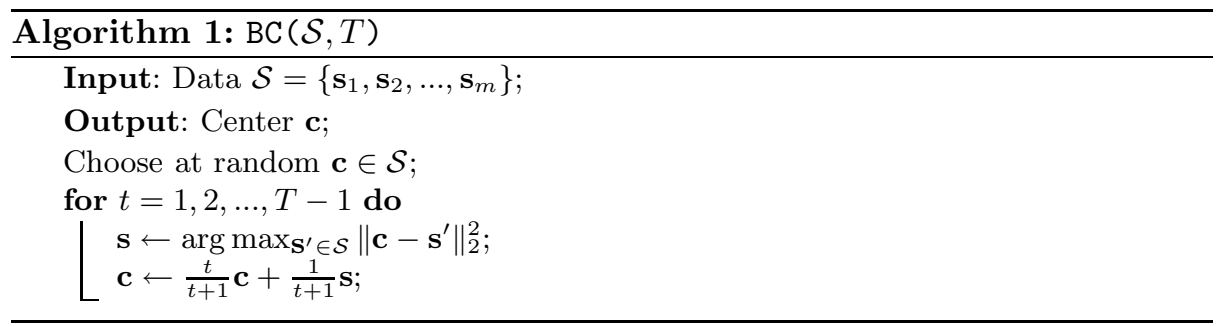

\section{Extending BC}

The primal SEBB problem is to find:

$$
\arg \min _{\mathbf{C}^{*}, r^{*}} r^{*} \text { s.t. } D_{F}\left(\mathbf{c}^{*}, \mathbf{s}_{i}\right) \leq r^{*}, \forall 1 \leq i \leq m
$$

Its Lagrangian is $L(\mathcal{S}, \boldsymbol{\alpha})=r^{*}-\sum_{i=1}^{m} \alpha_{i}\left(r^{*}-D_{F}\left(\mathbf{c}^{*}, \mathbf{s}_{i}\right)\right)$, with the additional Karush-Kuhn-Tucker condition $\boldsymbol{\alpha} \geq \mathbf{0}$. The solution to (5) is obtained by minimizing $L(\mathcal{S}, \boldsymbol{\alpha})$ for the parameters $\mathbf{c}^{*}$ and $r^{*}$, and then maximize the resulting dual for the Lagrange multipliers. We obtain $\partial L(\mathcal{S}, \boldsymbol{\alpha}) / \partial \mathbf{c}^{*}=\boldsymbol{\nabla}_{F}\left(\mathbf{c}^{*}\right) \sum_{i=1}^{m} \alpha_{i}-$ $\sum_{i=1}^{m} \alpha_{i} \boldsymbol{\nabla}_{F}\left(\mathbf{s}_{i}\right)$ and $\partial L(\mathcal{S}, \boldsymbol{\alpha}) / \partial r^{*}=1-\sum_{i=1}^{m} \alpha_{i}$. Setting $\partial L(\mathcal{S}, \boldsymbol{\alpha}) / \partial \mathbf{c}^{*}=\mathbf{0}$ and $\partial L(\mathcal{S}, \boldsymbol{\alpha}) / \partial r^{*}=0$ yields $\sum_{i=1}^{m} \alpha_{i}=1$ and:

$$
\mathbf{c}^{*}=\nabla_{F}^{-1}\left(\sum_{i=1}^{m} \alpha_{i} \nabla_{F}\left(\mathbf{s}_{i}\right)\right) .
$$

Table 1. Some common Bregman divergences and their associated functional averages. The second row depicts the general I (information) divergence, also known as KullbachLeibler (KL) divergence on the $d$-dimensional probability simplex. On the fourth row, $A$ is the inverse of the covariance matrix [2].

\begin{tabular}{llll}
\hline domain & $F(\mathbf{s})$ & $D_{F}(\mathbf{c}, \mathbf{s})$ & $c_{j}(1 \leq j \leq d)$ \\
\hline \hline $\mathbb{R}^{d}$ & $\sum_{j=1}^{d} s_{j}^{2}$ & $L_{2}^{2}$ norm & arithmetic mean \\
\hline$\left(\mathbb{R}^{+, *}\right)^{d}$ & & $(\mathrm{I} / \mathrm{KL})$-divergence & $\sum_{i=1}^{m} \alpha_{i} s_{i, j}$ \\
\hline$/ d$-simplex $\sum_{j=1}^{d} s_{j} \log s_{j}-s_{j} \sum_{j=1}^{d} c_{j} \log \left(c_{j} / s_{j}\right)-c_{j}+s_{j}$ & $\prod_{i=1}^{m} s_{i, j}^{\alpha_{i}}$ \\
\hline \multirow{2}{*}{$\left.\mathbb{R}^{+, *}\right)^{d}$} & $-\sum_{j=1}^{d} \log s_{j}$ & $\sum_{j=1}^{d}\left(c_{j} / s_{j}\right)-\log \left(c_{j} / s_{j}\right)-1$ & $1 / \sum_{i=1}^{m}\left(\alpha_{i} / s_{i, j}\right)$ \\
\hline $\mathbb{R}^{d}$ & $\mathbf{s}^{T} A \mathbf{s}$ & Mahalanobis distance & arithmetic mean \\
\hline & $p \in \mathbb{N} \backslash\{0,1\}$ & $(\mathbf{c}-\mathbf{s})^{T} A(\mathbf{c}-\mathbf{s})$ & $\sum_{i=1}^{m} \alpha_{i} s_{i, j}$ \\
$\mathbb{R}^{d} / \mathbb{R}^{+d}$ & $(1 / p) \sum_{j=1}^{d} s_{j}^{p}$ & $\sum_{j=1}^{d} \frac{c_{j}^{p}}{p}+\frac{(p-1) s_{j}^{p}}{p}-c_{j} s_{j}^{p-1}$ & $\left(\sum_{i=1}^{m} \alpha_{i} s_{i, j}^{p-1}\right)^{1 /(p-1)}$ \\
\hline \hline
\end{tabular}


Because $F$ is strictly convex, $\nabla_{F}$ is bijective, and $\mathbf{c}^{*}$ lies in the convex closure of $\mathcal{S}$. Finally, we are left with finding:

$$
\arg \max _{\boldsymbol{\alpha}} \sum_{i=1}^{m} \alpha_{i} D_{F}\left(\boldsymbol{\nabla}_{F}^{-1}\left(\sum_{j=1}^{m} \alpha_{j} \boldsymbol{\nabla}_{F}\left(\mathbf{s}_{j}\right)\right), \mathbf{s}_{i}\right) \text { s.t. } \boldsymbol{\alpha} \geq \mathbf{0}, \sum_{i=1}^{m} \alpha_{i}=1 .
$$

This problem generalizes the dual of support vector machines: whenever $F(\mathbf{s})=$ $\sum_{i=1}^{d} s_{i}^{2}=\langle\mathbf{s}, \mathbf{s}\rangle$ (Table 1), we return to their kernel-based formulation 4]. There are essentially two categories of Lagrange multipliers in vector $\boldsymbol{\alpha}$. Those corresponding to points of $\mathcal{S}$ lying on the interior of $\mathcal{B}_{\mathbf{C}^{*}, r^{*}}$ are zero, since these points satisfy their respective constraints. The others, corresponding to the support points of the ball, are strictly positive. Each $\alpha_{i}>0$ represents the contribution of its support point to the computation of the circumcenter of the ball. Eq. (6) is thus some functional average of the support points of the ball, to compute $\mathbf{c}^{*}$.

\subsection{The Modified Bădoiu-Clarkson Algorithm, MBC}

There is more on eq. (6). A Bregman divergence is not affected by linear terms: $D_{F+q}=D_{F}$ for any constant $q[6]$. Thus, the partial derivatives of $F$ in $\nabla_{F}($.$) de-$ termine entirely the Bregman divergence. The following Lemma is then immediate.

Lemma 2. The set of functional averages (6) is in bijection with the set of Bregman divergences (1).

The connection between the functional averages and divergences is much interesting because the classical means commonly used in many domains, such as convex analysis, parametric estimation, signal processing, are valid examples of functional averages. A nontrivial consequence of Lemma 2 is that each of them encodes the SEBB solution for an associated Bregman divergence. Apart from the SEBB problem, this is interesting because means are popular statistics, and we give a way to favor the choice of a mean against another one depending on the domain of the data and its "natural" distortion measure. Table 1 presents some Bregman divergences and their associated functional averages, for the most commonly encountered.

Speaking of bijections, previous results showed the existence of a bijection between Bregman divergences and the family of exponential distributions [2]. This has helped the authors to devise a generalization of the $k$-means algorithm. In our case, Lemma 2 is also of some help to generalize BC. Clearly, the dual problem in eq. (7) does not admit the convenient representation of SVMs, and it seems somehow hard to use a kernel trick replacing the elements of $\mathcal{S}$ by local transformations involving $F$ prior to solving problem (17). However, the dual suggests a very simple algorithm to approximate $\mathbf{c}^{*}$, which consists in making the parallel between $\boldsymbol{\nabla}\left(\mathbf{c}^{*}\right)=\sum_{i=1}^{m} \alpha_{i} \boldsymbol{\nabla}_{F}\left(\mathbf{s}_{i}\right)$ (6) and the arithmetic mean in Table 1] and consider (6) as the solution to a minimum distortion problem involving gradients into a $L_{2}^{2}$ space. We can thus seek:

$$
\arg \min _{\mathbf{g}^{*}, r^{\prime *}} r^{\prime *} \text { s.t. }\left\|\mathbf{g}^{*}-\nabla_{F}\left(\mathbf{s}_{i}\right)\right\|_{2}^{2} \leq r^{*}, \forall 1 \leq i \leq m .
$$


Finally, approximating (5) amounts to running the so-called Modified BădoiuClarkson algorithm in the gradient space, MBC . Because $\nabla_{F}$ is bijective, this is guaranteed to yield a solution. The remaining question is whether $\boldsymbol{\nabla}_{F}^{-1}(\mathbf{g})=\mathbf{c}$ is close enough from the solution $\mathbf{c}^{*}$ of (5). The following Lemma upperbounds the sum of the two divergences between $\mathbf{c}$ and any point of $\mathcal{S}$, as a function of $r^{*}$. It shows that the two centers can be very close to each other; in fact, they can be much closer than with a naive application of Bădoiu-Clarkson directly in $\mathcal{S}$. The Lemma makes the hypothesis that the Hessian of $F, H_{F}$, is non singular. As a matter of fact, it is diagonal (without zero in the diagonal) for all classical examples of Bregman divergences, see Table 1, so this is not a restriction either. In the Lemma, we let $f$ denote the minimal non zero value of the Hessian norm inside the convex closure of $\mathcal{S}: f=\min _{\mathbf{X} \in \operatorname{co}(\mathcal{S}):\left\|H_{F}(\mathbf{x})\right\|_{2}>0}\left\|H_{F}(\mathbf{x})\right\|_{2}$.

Lemma 3. $\forall s \in \mathcal{S}$, we have:

$$
D_{F}\left(s, \nabla_{F}^{-1}(\boldsymbol{g})\right)+D_{F}\left(\boldsymbol{\nabla}_{F}^{-1}(\boldsymbol{g}), \boldsymbol{s}\right) \leq(1+\varepsilon)^{2} r^{*} / f,
$$

where $\boldsymbol{g}=B C\left(\left\{\nabla_{F}\left(\boldsymbol{s}_{i}\right): \boldsymbol{s}_{i} \in \mathcal{S}\right\}, T\right), r^{*}$ is defined in eq. (8)), and $\varepsilon$ is the error parameter of $B C$.

(proof omitted due to the lack of space) Remark that Lemma 3 is optimal, in the sense that if we consider $D_{F}=L_{2}^{2}$, then each point $\mathbf{s}_{i} \in \mathcal{S}$ becomes $2 \mathbf{s}_{i}$ in $\mathcal{S}^{\prime}$. The optimal radii in (5) and (8) satisfy $r^{*}=4 r^{*}$, and we have $f=2$. Plugging this altogether in eq. (9) yields $2\|\mathbf{c}-\mathbf{s}\|_{2}^{2} \leq(1+\varepsilon)^{2} \times 4 r^{*} / 2$, i.e. $\|\mathbf{c}-\mathbf{s}\|_{2} \leq(1+\varepsilon) \sqrt{r^{*}}$, which is exactly Bădiou-Clarkson's bound [1] (here, we have fixed $\mathbf{c}=\boldsymbol{\nabla}_{F}^{-1}(\mathbf{g})$, like in Lemma 3). Remark also that Lemma 3 upperbounds the sum of both possible divergences, which is very convenient given the possible asymmetry of $D_{F}$.

\subsection{The Bregman-Bădoiu-Clarkson Algorithm, BBC}

It is straightforward to check that at the end of BC (algorithm 1), the following holds true:

$$
\left\{\begin{array}{l}
\mathbf{c}=\sum_{i=1}^{m} \hat{\alpha}_{i} \mathbf{s}_{i}, \sum_{i=1}^{m} \hat{\alpha}_{i}=1, \hat{\boldsymbol{\alpha}} \geq \mathbf{0}, \\
\forall 1 \leq i \leq m, \hat{\alpha}_{i} \neq 0 \text { iff } \mathbf{s}_{i} \text { is chosen at least once in BC } .
\end{array}\right.
$$

Since the furthest points chosen by BC ideally belong to $\partial \mathcal{B}_{\mathbf{C}^{*}, r^{*}}$, and the final expression of $\mathbf{c}$ matches the arithmetic average of Table 1 it comes that BC directly tackles an iterative approximation of eq. (6) for the $L_{2}^{2}$ Bregman divergence. If we replace $L_{2}^{2}$ by an arbitrary Bregman divergence, then BC can be generalized in a quite natural way to algorithm BBC (for Bregman-Bădoiu-Clarkson) below. Again, it is straightforward to check that at the end of BBC, we have generalized the iterative approximation of $\mathrm{BC}$ to eq. (6) for any Bregman divergence, as we have:

$$
\left\{\begin{array}{l}
\mathbf{c}=\nabla_{F}^{-1}\left(\sum_{i=1}^{m} \hat{\alpha}_{i} \nabla_{F}\left(\mathbf{s}_{i}\right)\right), \sum_{i=1}^{m} \hat{\alpha}_{i}=1, \hat{\boldsymbol{\alpha}} \geq \mathbf{0} \\
\forall 1 \leq i \leq m, \hat{\alpha}_{i} \neq 0 \text { iff } \mathbf{s}_{i} \text { is chosen at least once in BC }
\end{array}\right.
$$




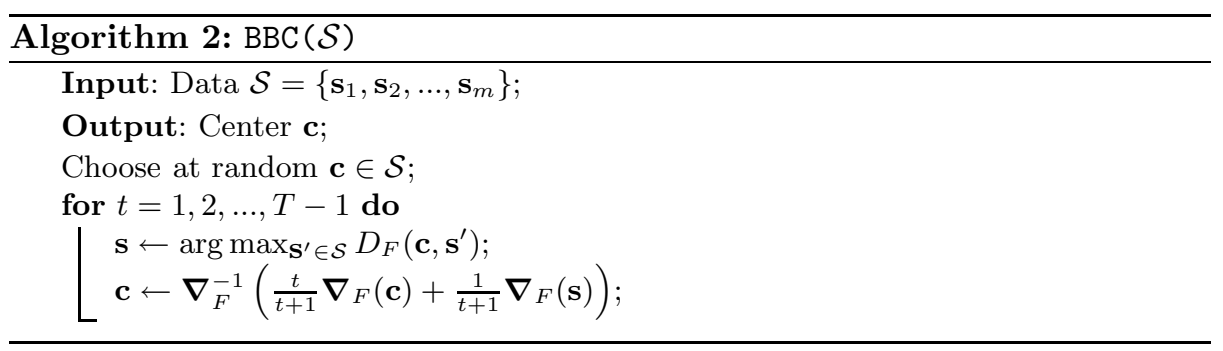

The main point is whether $\hat{\boldsymbol{\alpha}}$ is a good approximation to the true vector of Lagrange multipliers $\boldsymbol{\alpha}$. From the theoretical standpoint, the proof of BC's approximation ratio becomes tricky when lifted from $L_{2}^{2}$ to an arbitrary Bregman divergence, but it can be shown that many of the key properties of the initial proof remain true in this more general setting. An experimental hint that speaks for itself for the existence of such a good approximation ratio is given in the next Section.

\section{Experimental Results}

Due to the lack of space, we only present results on BBC . To evaluate the quality of the approximation of $\mathrm{BBC}$ for the SEBB, we have ran the algorithm for three popular representative Bregman divergences. For each of them, averages over a hundred runs were performed for $T=200$ center updates (see algorithm 2). In each run, a random Bregman ball is generated, and $\mathcal{S}$ is sampled uniformly at random in the ball. Since we know the SEBB, we have a precise idea of the quality of the approximation found by BBC on the SEBB. Figure 2 gives a synthesis of the results for $d=2$. [1]'s bound is plotted for each divergence,



$1.02 \pm 0.16$

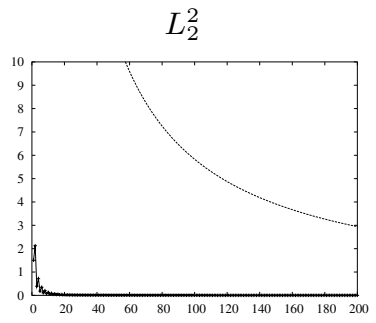

$1.03 \pm 0.19$
Kullbach-Leibler

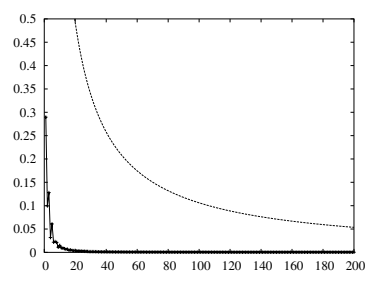

$1.06 \pm 0.17$

Fig. 2. Average approximation curves for 100 runs of BBC algorithm for three Bregman divergences: Itakura-Saito, $L_{2}^{2}$ and $\mathrm{KL}(d=2, m=1000, T=200)$. The dashed curves are Bădoiu-Clarkson's error bound as a function of the iteration number $t$, and the bottom, plain curves, depict $\left(D_{F}\left(\mathbf{c}^{*}, \mathbf{c}\right)+D_{F}\left(\mathbf{c}, \mathbf{c}^{*}\right)\right) / 2$ as a function of $t$ for each divergence, where $\mathbf{c}$ is the output of $\mathrm{BBC}$ and $\mathbf{c}^{*}$ is the optimal center. The bottom number depict the estimated error $(\%) \pm$ standard deviation. 
Table 2. Estimated errors for the SEBB problem for data generated using a mixture of $u$ gaussians, for $u=1,3,5,10,20$. Conventions and parameters follow Figure 2

\begin{tabular}{cccc}
$u$ & Itakura-Saito & $L_{2}^{2}$ & Kullbach-Leibler \\
\hline $\mathbf{1}$ & $0.37 \pm 0.06$ & $0.43 \pm 0.09$ & $0.39 \pm 0.08$ \\
$\mathbf{3}$ & $0.40 \pm 0.04$ & $0.41 \pm 0.10$ & $0.41 \pm 0.06$ \\
$\mathbf{5}$ & $0.41 \pm 0.04$ & $0.43 \pm 0.10$ & $0.41 \pm 0.04$ \\
$\mathbf{1 0}$ & $0.40 \pm 0.02$ & $0.44 \pm 0.09$ & $0.42 \pm 0.05$ \\
$\mathbf{2 0}$ & $0.41 \pm 0.02$ & $0.43 \pm 0.08$ & $0.41 \pm 0.04$ \\
\hline
\end{tabular}

even when it holds formally only for $L_{2}^{2}$. The other two curves give an indication of the way this bound behaves with respect to the experimental results. It is easy to see that for each divergence, there is a very fast convergence of the center found, c, to the optimal center $\mathbf{c}^{*}$. Furthermore, the experimental divergences are always much smaller than [1]'s bound, for each divergence (very often by a factor 100 or more). We have checked this phenomenon for higher dimensions, up to $d=20$. Following [7, the errors given are the ratio of the number of support points over the whole number of points. A good method would typically select a very small number of points, regardless of the domain. While this is clearly displayed in Figure 2, Table2 goes deeper in this phenomenon, as it displays the errors when the points are drawn from random mixtures of Gaussians. Even in this case, where the Gaussians may be very distant from each other, MBC with the three Bregman divergences of Figure 2 still displays a very low error.

\section{References}

1. M. Bădoiu and K.-L. Clarkson. Optimal core-sets for balls, 2002. Manuscript.

2. A. Banerjee, S. Merugu, I. Dhillon, and J. Ghosh. Clustering with bregman divergences. In Proc. of the $4^{\text {th }}$ SIAM International Conference on Data Mining, pages 234-245, 2004.

3. L. M. Bregman. The relaxation method of finding the common point of convex sets and its application to the solution of problems in convex programming. USSR Comp. Math. and Math. Phys., 7:200-217, 1967.

4. C. J. C. Burges. A tutorial on support vector machines for pattern recognition. Data Mining and Knowledge Discovery, 2:121-167, 1998.

5. K. Crammer and G. Chechik. A needle in a haystack: local one-class optimization. In Proc. of the $21^{\text {th }}$ International Conference on Machine Learning, 2004.

6. C. Gentile and M. Warmuth. Proving relative loss bounds for on-line learning algorithms using Bregman divergences. In Tutorials of the $13^{\text {th }}$ International Conference on Computational Learning Theory, 2000.

7. D. Tax and R. Duin. Support Vector Domain Description. Pattern Recognition Letters, 20:1191-1199, 1999.

8. I. W. Tsang, J. T. Kwok, and P.-M. Cheung. Core Vector Machines: fast SVM training on very large datasets. Journal of Machine Learning Research, 6:363-392, 2005 . 